\title{
New Results from the Daya Bay Reactor Neutrino Experiment
}

\author{
Dmitry V. Naumov ${ }^{* \dagger}$ \\ Joint Institute for Nuclear Research, Dubna \\ E-mail: dmitryvnaumov@gmail.com
}

Some recent results obtained by Daya Bay Collaboration and presented at Neutrino Telescopes 2017 are briefly reviewed. The most precise measurement of neutrino mixing $\sin ^{2} 2 \theta_{13}=0.0841 \pm 0.0027$ (stat.) \pm 0.0019 (syst.) and mass squared difference $\left|\Delta m_{\text {ee }}^{2}\right|=$ $[2.50 \pm 0.06$ (stat.) \pm 0.06 (syst.) $] \times 10^{-3} \mathrm{eV}^{2}$ was obtained using $\mathrm{nGd}$ data set. These results agree with an independent analysis of $\mathrm{nH}$ data sample.

The data was analized also within the model of neutrino wave packet. For the first time an upper limit on the intrinsic relative dispersion of neutrino momentum $\sigma_{\text {rel }}<0.20$ at a $95 \%$ C.L. was obtained. The allowed decoherence effect due to the wave packet nature of neutrino oscillation is found to be insignificant for reactor antineutrinos detected by the Daya Bay experiment thus ensuring an unbiased measurement of the oscillation parameters $\sin ^{2} 2 \theta_{13}$ and $\Delta m_{32}^{2}$ within the plane wave model.

The flux of reactor $\bar{v}_{e}$ was measured and found to be in agreement with old reactor experiments observing $\bar{v}_{e}$ flux smaller than expectation in Huber+Muller model. Comparing observed and predicted energy spectra we find an almost flat deficit of measured events for $E_{v}<5 \mathrm{MeV}$ and an agreement with the Huber+Muller model for $5.5 \mathrm{MeV} \lesssim E_{V} \lesssim 6.5 \mathrm{MeV}$.

A possible hypothesis of the observed flux deficit invokes $\bar{v}_{e}$ oscillation into a sterile state. This hypothesis was excluded for a large range of $\Delta m_{41}^{2}$. A combined analysis of Daya Bay, Bugey-3 and MINOS data excludes most of the allowed by LSND and MiniBooNE experiments sterileneutrino phase space for $\Delta m_{41}^{2}<0.8 \mathrm{eV}^{2}$ at $90 \%$ C.L.

XVII International Workshop on Neutrino Telescopes

13-17 March 2017

Venezia, Italy

\footnotetext{
*Speaker.

$\dagger^{\dagger}$ on behalf of Daya Bay Collaboration
} 


\section{Introduction}

Nuclear reactors provide an intensive source of electron anti-neutrino $\bar{v}_{e}$. Each GW of reactor thermal power corresponds to about $10^{20}$ of $\bar{v}_{e}$ emitted isotropically every second. Detection of $\bar{v}_{e}$ from nuclear reactor accurately reconstructing its energy provides a unique tool allowing to study properties of neutrinos, precise details of nuclear fuel burning and perform other measurements [1].

The Daya Bay Reactor Neutrino Experiment began data taking in 2011 and soon after discovered a non-zero value of lepton mixing angle $\theta_{13}$ [2]. Since then, the Daya Bay Collaboration collected a record number exceeding $2.5 \cdot 10^{6}$ of $\bar{v}_{e}+p \rightarrow e^{+}+n$ interactions (inverse $\beta$ decays, "IBD" in what follows) allowing to perform important physical analyses. In this note we briefly review some of the most recent results.

\section{Daya Bay Experiment}

The Daya Bay experiment is composed of two near underground experimental halls (EH1 and $\mathrm{EH} 2$ ) and one far underground hall (EH3). Each of the experimental halls hosts identically designed antineutrino detectors (ADs). EH1 and EH2 contain two ADs each, while EH3 contains four ADs. The Gd-doped liquid scintillator target is a cylinder of three meters in both height and diameter.

Electron antineutrinos are produced in three pairs of nuclear reactors via $\beta$ decays of neutronrich daughters of the fission isotopes ${ }^{235} \mathrm{U},{ }^{238} \mathrm{U},{ }^{239} \mathrm{Pu}$ and ${ }^{241} \mathrm{Pu}$. The coincidence of the prompt $\left(e^{+}\right.$ionization and annihilation) and delayed ( $n$ capture on $\mathrm{Gd}$ ) signals efficiently suppresses the backgrounds, which amounted to less than $1.5 \%(2.5 \%)$ of the IBD candidates in the near (far) halls [12]. The IBD detection efficiency is $80.6 \%$ with uncertainty correlated (uncorrelated) between detectors $1.93 \%(0.13 \%)$. The uncertainty correlated between detectors is essentially canceled in the oscillation analysis.

The detectors have a light yield of about 165 photoelectrons/MeV and a reconstructed energy resolution $\delta_{E} / E \approx 8 \%$ at $1 \mathrm{MeV}$ of deposited energy in the scintillator. More details on the experimental setup are contained in Refs. [3, 4, 5, 6].

First 217 days the Daya Bay experiment was taking data with six ADs - two in EH1, one in $\mathrm{EH} 2$ and three in EH3. Several analyses of these dataset were published [7, 8, 9, 10].

During summer 2012 shutdown two more AD were installed and since then the experiment is taking data according to its designed eight AD's configuration. After 621 days the collected number of IBD events exceeded $10^{6}$ and some analyses updated their results in Refs. [3, 11].

The most recent results are based on 1230 days of data taking [12]. In what follows we will discuss results based on these three datasets indicating the number of days (217, 621 and 1230 days).

\section{Precision measurement of $\theta_{13}$ and $\Delta m_{32}^{2}$}

\subsection{Results from nGd analysis based on 1230 days}

Comparing ratio of observed number of IBD events to prediction assuming no neutrino oscillation results in a deficit of observed $\bar{v}_{e}$ interactions in the far detector with respect to near detectors 
as be seen from Fig. 1. Assuming neutrino oscillation allowed to fit the ratio with

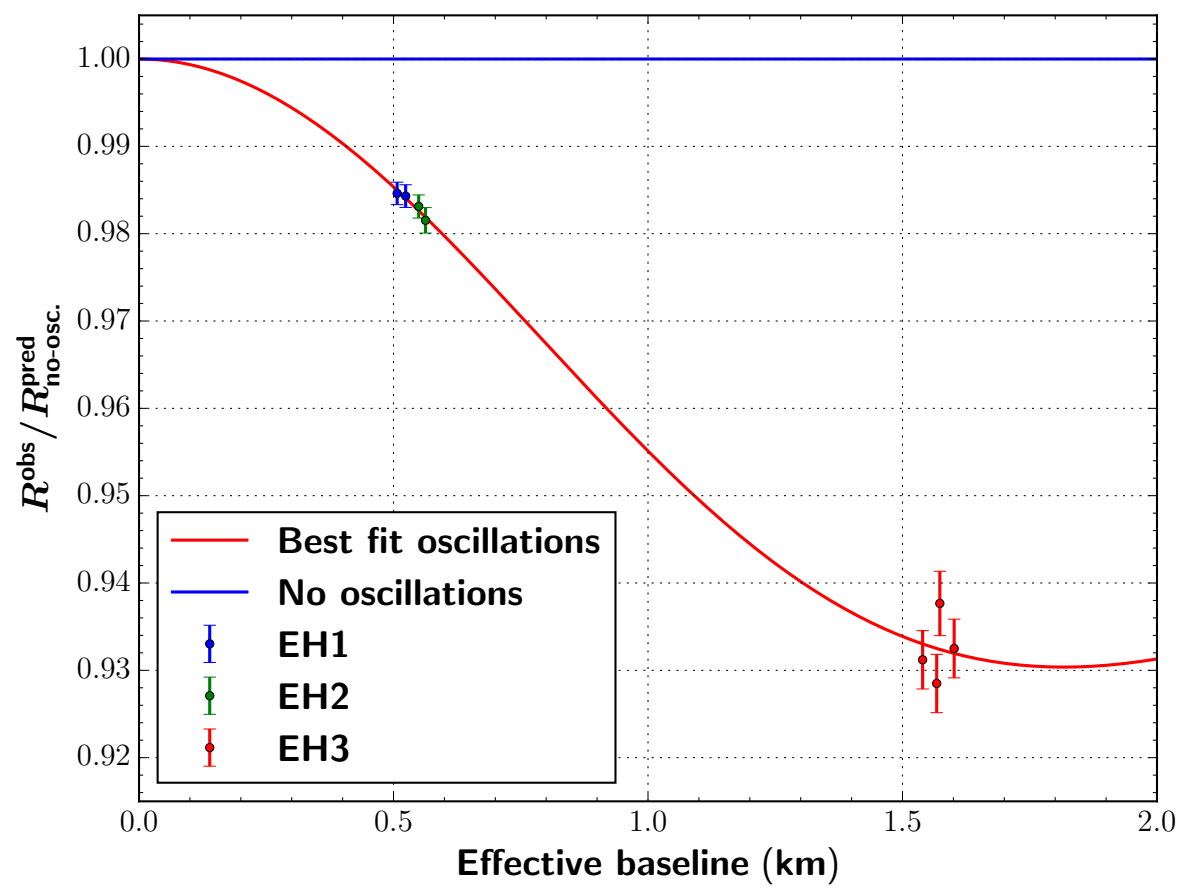

Figure 1: Ratio of observed number of events to prediction assuming no neutrino oscillation as a function of effective baseline. Extrapolation of the model to a baseline of zero determined the absolute normalization of the reactor $\bar{v}_{e}$ flux, $R^{\text {pred }}(L=0)$. The points representing the near (far) detectors are displaced by $\pm 6 \mathrm{~m}$ $( \pm 30 \mathrm{~m}$ ) for clarity. The blue (red) line corresponds to the ratio assuming no (best-fit) neutrino oscillation hypothesis.

$$
\begin{aligned}
\sin ^{2} 2 \theta_{13} & =0.0850 \pm 0.0030 \text { (stat.) } \pm 0.0028 \text { (syst.) } \\
\frac{\chi^{2}}{\mathrm{NDF}} & =\frac{5.07}{8-2}=0.85
\end{aligned}
$$

using $\left|\Delta m_{32}^{2}\right|=(2.43 \pm 0.07) \times 10^{-3} \mathrm{eV}^{2}$ as input [13]

Neutrino oscillation model is also in best agreement with the observed energy spectra as can be seen in Fig. 2. The corresponding parameters of the three-flavor model read

$$
\begin{aligned}
\sin ^{2} 2 \theta_{13} & =0.0841 \pm 0.0027 \text { (stat.) } \pm 0.0019 \text { (syst.) } \\
\left|\Delta m_{\mathrm{ee}}^{2}\right| & =[2.50 \pm 0.06 \text { (stat.) } \pm 0.06 \text { (syst.) }] \times 10^{-3} \mathrm{eV}^{2}, \\
\Delta m_{32}^{2}(\mathrm{NH}) & =[2.45 \pm 0.06 \text { (stat.) } \pm 0.06 \text { (syst.) }] \times 10^{-3} \mathrm{eV}^{2} \\
\Delta m_{32}^{2}(\mathrm{IH}) & =-[2.56 \pm 0.06 \text { (stat.) } \pm 0.06 \text { (syst.) }] \times 10^{-3} \mathrm{eV}^{2} \\
\frac{\chi^{2}}{\mathrm{NDF}} & =\frac{234.7}{280-17}=0.89 .
\end{aligned}
$$

The observed spectral deficit of reactor $\bar{v}_{e}$ can be displayed as a function of $L_{\mathrm{eff}} / E_{v}$, where $L_{\mathrm{eff}}$ is the effective baseline between a given detector and the six reactors. Fig. 3 illustrates the ratio of the observed $\bar{v}_{e}$ signal over the no-oscillation prediction versus the effective baseline for all eight 


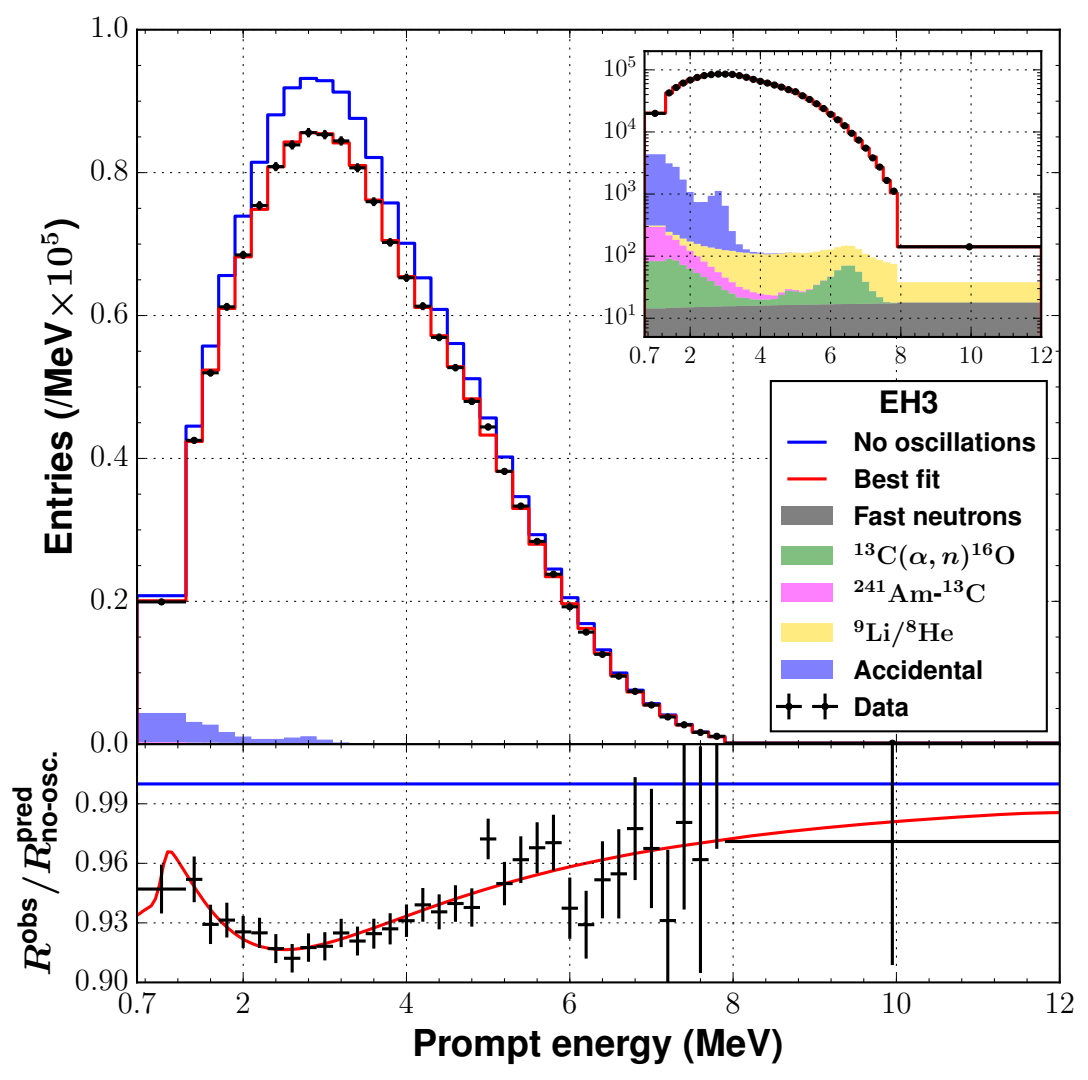

Figure 2: Reconstructed positron energy spectra for the $\bar{v}_{e}$ candidate interactions in EH3 (black points). The measurements are compared with the prediction assuming no oscillation (blue line) and the best-fit threeflavor neutrino oscillation model (red line). The inset in semi-logarithmic scale shows the backgrounds. The ratio of the background-subtracted spectra to prediction assuming no oscillation is shown in the panel beneath energy spectrum.

detectors. The measurement sampled $\bar{v}_{e}$ survival over almost one full cycle, demonstrating distinct evidence in support of neutrino flavor oscillation.

\subsection{Results from $\mathrm{nH}$ analysis based on 621 days}

Capture of recoil neutron from IBD on a hydrogen $n H$-analysis provides an additional way to detect reactor $\bar{v}_{e}$. This type of analysis has an increased IBD events statistics due to additional mass of the liquid scintillator (plus 20 tons for each AD), and largely independent systematical uncertainties. nH-data has larger contribution from accidental background because of a lower delayed energy $(\simeq 2.2 \mathrm{MeV})$. An analysis of $\mathrm{nH}$-data also displayed a significant rate deficit and the energy spectral distortion both consistent with neutrino oscillation [11]. The best-fit value

$$
\sin ^{2} 2 \theta_{13}=0.071 \pm 0.011
$$

is the third world precise measurement after Daya Bay (nGd) [12] and RENO (nGd) [14]. 


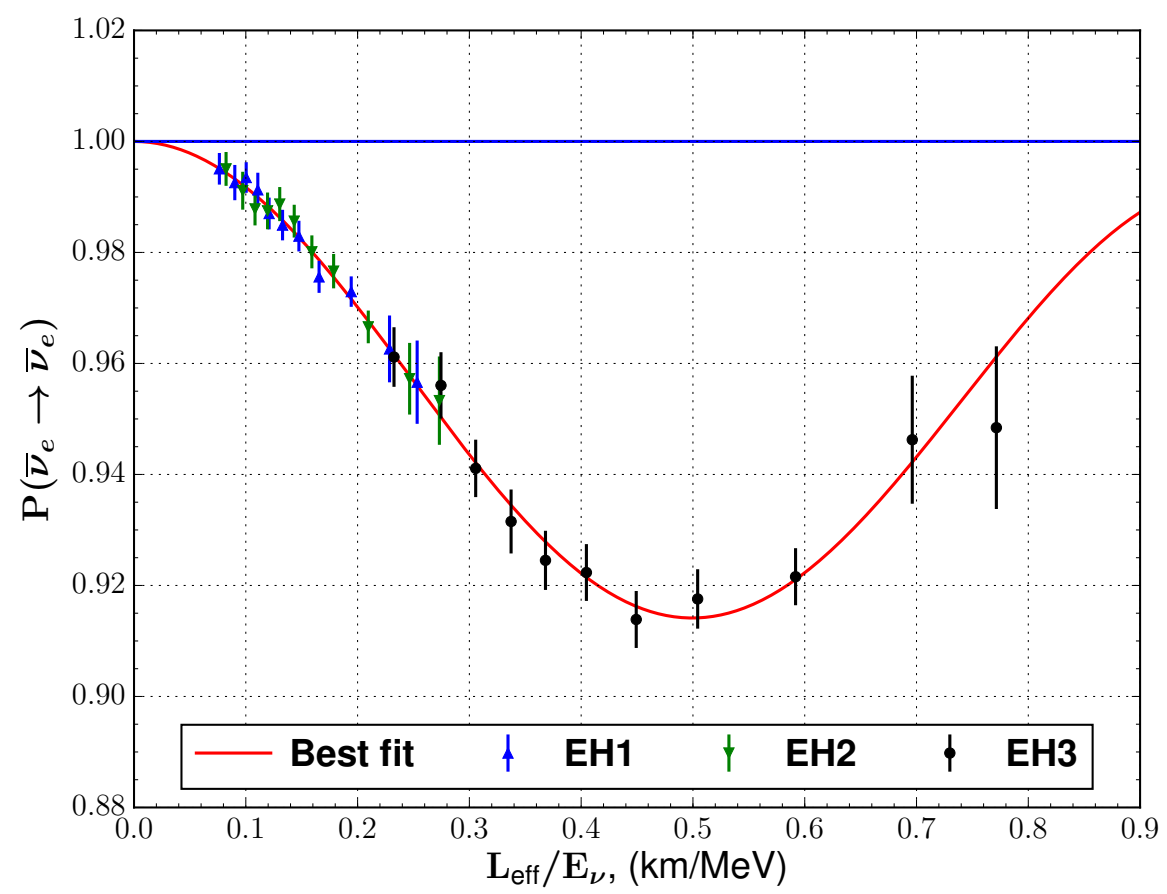

Figure 3: Measured reactor $\bar{v}_{e}$ spectral distortion, displayed as the oscillation survival probability versus $L_{\text {eff }} / E_{v}$.

\section{Study of wave packet effects in neutrino oscillations}

The results reported in Sec. 3 were obtained assuming plane wave model for neutrino. The plane wave model is known to be not self-consistent [15]. Models free from internal inconsistencies describe neutrino as a wave packet (see, for example Refs. [16, 17, 18] and references therein).

The Daya Bay data set corresponding to 621 days of data taking was fitted within a wave packet model with one more free parameter $\sigma_{\text {rel }}=\sigma_{p} / p$, where $\sigma_{p}$ is momentum dispersion of neutrino wave packet. The allowed regions of $\left(\Delta m_{32}^{2}, \sin ^{2} 2 \theta_{13}\right.$ vs $\left.\sigma_{\text {rel }}\right)$ are displayed in Fig. 4 . For the values of $\sigma_{\text {rel }} \lesssim 10^{-16}$ the decoherence effects lead to strong correlations between $\Delta m_{32}^{2}, \sin ^{2} 2 \theta_{13}$ and $\sigma_{\text {rel }}$, yielding smaller values of $\Delta m_{32}^{2}$ and larger values of $\sin ^{2} 2 \theta_{13}$. However such small values of $\sigma_{\text {rel }}$ are excluded considering actual dimensions of the reactor cores and detectors $\sigma_{x} \lesssim 2 \mathrm{~m}$ which can be translated into $\sigma_{\text {rel }} \gtrsim 10^{-14}$.

The reported analysis of the Daya Bay data provides, for the first time, an allowed interval of the intrinsic relative dispersion of neutrino momentum $2.38 \cdot 10^{-17}<\sigma_{\text {rel }}<0.23$ or taking into account the actual dimensions of the reactor cores and detectors $\sigma_{\text {rel }}<0.20$ at a $95 \%$ C.L. This upper limit of $\sigma_{\text {rel }}$ implies that $\sigma_{x} \gtrsim 10^{-11} \mathrm{~cm}$ exceeds size of any nucleus thus excluding a theoretical possibility of neutrino wave function to be formed at nuclear scales.

\section{Reactor $\bar{v}_{e}$ flux and energy spectrum measurement}

Total number of IBD events collected during 217 days of data taking are compared to expectations using two models of reactor $\bar{v}_{e}$ flux calculations: Huber+Mueller model $[19,20]$ and 


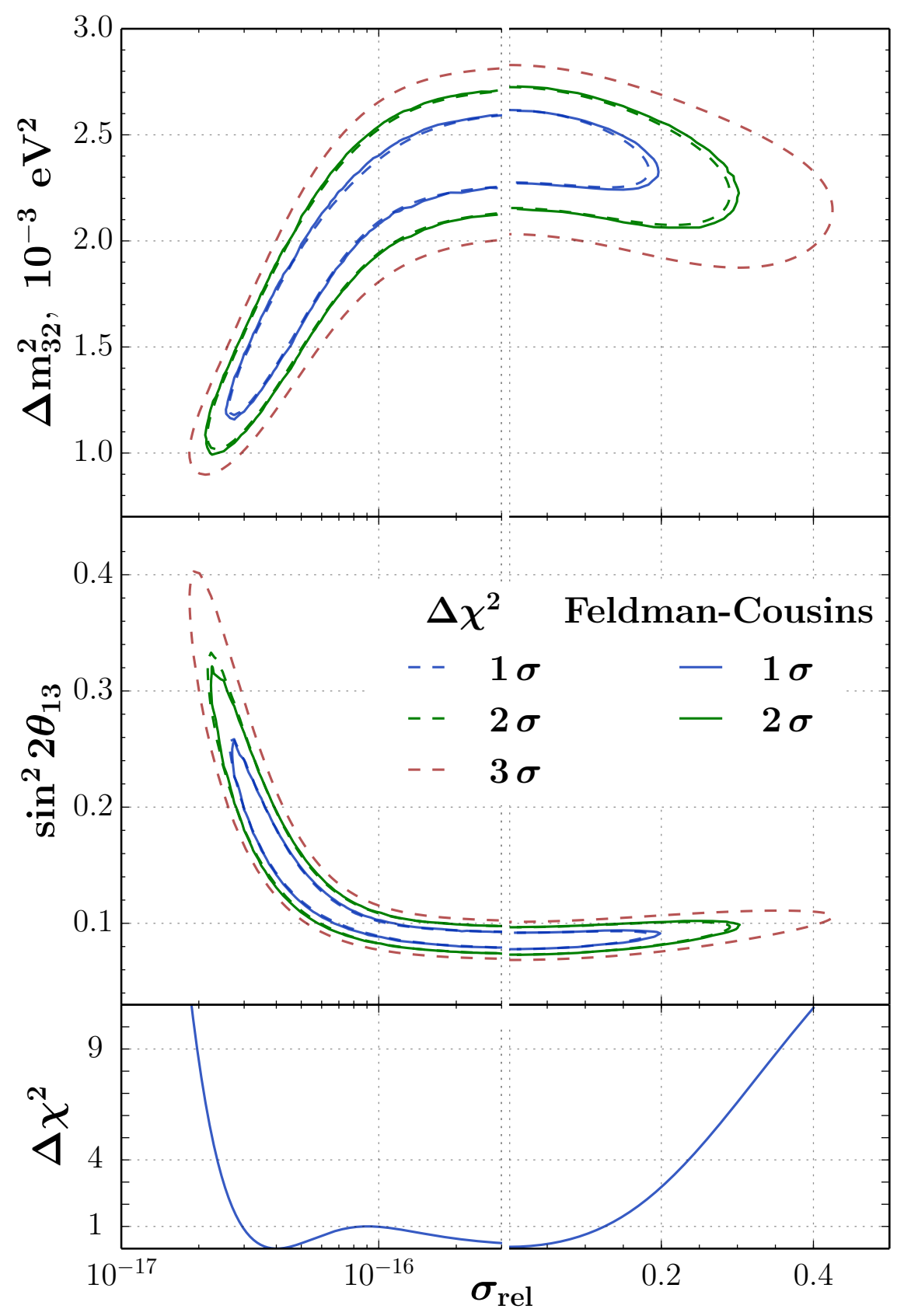

Figure 4: Allowed regions of $\left(\Delta m_{32}^{2}, \sigma_{\text {rel }}\right)$ (top) and of $\left(\sin ^{2} 2 \theta_{13}, \sigma_{\text {rel }}\right)$ (middle) parameters obtained with fixed-level $\Delta \chi^{2}$ (contours corresponding to $1 \sigma, 2 \sigma, 3 \sigma$ C.L., dashed lines) and within the Feldman-Cousins (contours corresponding to $1 \sigma, 2 \sigma$ C.L., solid lines) methods. Bottom panel shows the marginalized $\Delta \chi^{2}\left(\sigma_{\text {rel }}\right)$ statistic vs $\sigma_{\text {rel }}$. Note the break in the abscissa and the change from a logarithmic to linear scale.

ILL+Vogel model [21], accounting for neutrino oscillation and detector response. The observed 
Data/Expectation ratio $(R)$ reads

$$
\begin{aligned}
\mathrm{R}(\text { Huber }+ \text { Mueller }) & =0.946 \pm 0.022 \\
\mathrm{R}(\mathrm{ILL}+\text { Vogel }) & =0.991 \pm 0.023 .
\end{aligned}
$$

The observed disagreement with Huber+Mueller model is consistent with results of previous experiments with reactor $\bar{v}_{e}$ performed at baselines shorter than $100 \mathrm{~m}$ as can be seen from Fig. 5 . The global world average of $R$ is found to be $R=0.943 \pm 0.008$ (exp) \pm 0.025 (model).

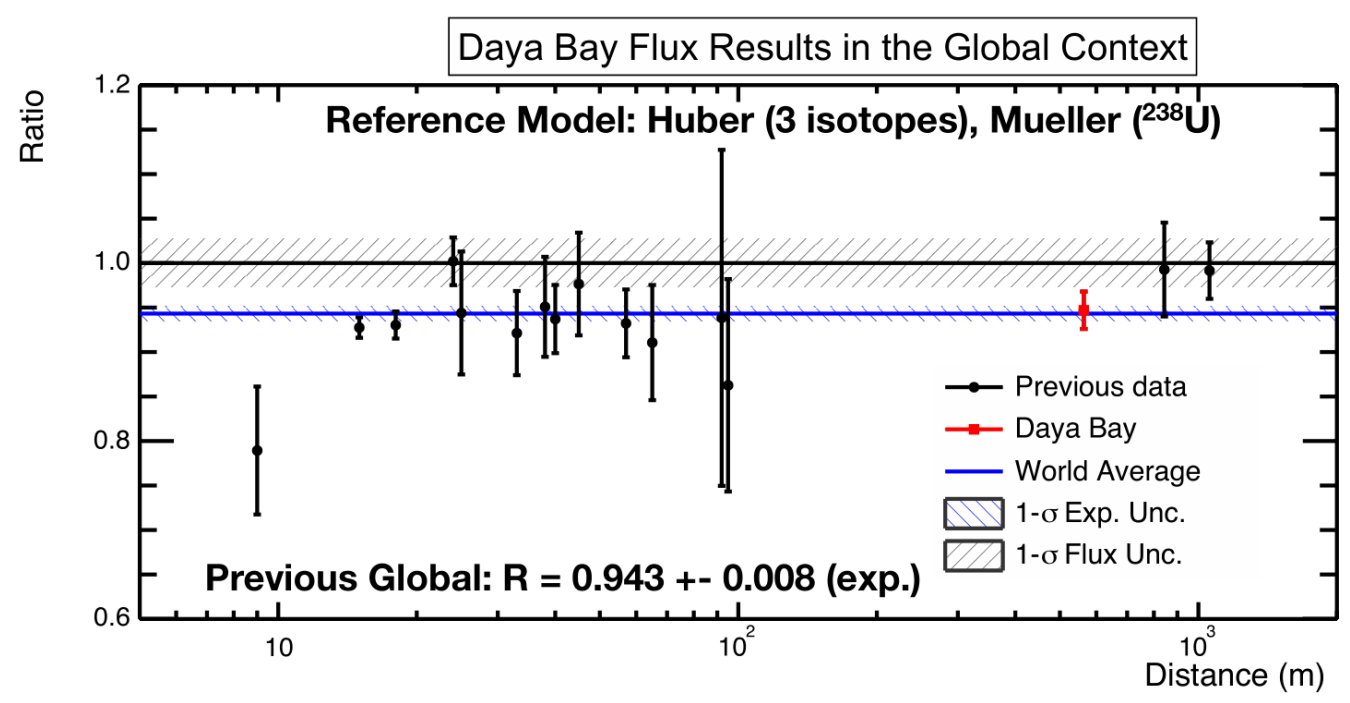

Figure 5: The measured reactor $\bar{v}_{e}$ rate as a function of the distance from the reactor, normalized to the theoretical prediction using the Huber+Mueller model. The rate is corrected for 3-flavor neutrino oscillations at each baseline. The blue shaded region represents the global average and its $1 \sigma$ uncertainty. The $2.7 \%$ model uncertainty is shown as a band around unity. Measurements at the same baseline are combined for clarity. The Daya Bay measurement is shown at the flux-weighted baseline $(573 \mathrm{~m})$ of the two near halls

A comparison of measured neutrino energy spectra with calculations using Huber+Mueller model is shown in Fig. 6. One can see an almost flat deficit of measured events for $E_{v}<5 \mathrm{MeV}$ and an agreement with the model for $5.5 \mathrm{MeV} \lesssim E_{v} \lesssim 6.5 \mathrm{MeV}$. The latter is often known in the literature as the "bump". We find this terminology a bit misleading because the "bump" appears in the energy region of formal agreement between measurement and model after the overall normalization is applied.

\section{Search for sterile neutrino admixture in $v_{e}$ state}

A number of results in neutrino physics can not be understood as due to three neutrino oscillations [22, 23, 24, 25, 26, 27]. Sterile neutrino was a popular hypothesis to explain above mentioned anomalies in neutrino physics.

Sterile neutrino is a coherent superposition of mass eigenstates $\left(v_{i}, i \geq 4\right)$ whose interaction amplitude with $W^{ \pm}$and $Z$ bosons is nearly zero (see, for example, a discussion in Ref. [28] and references therein). 


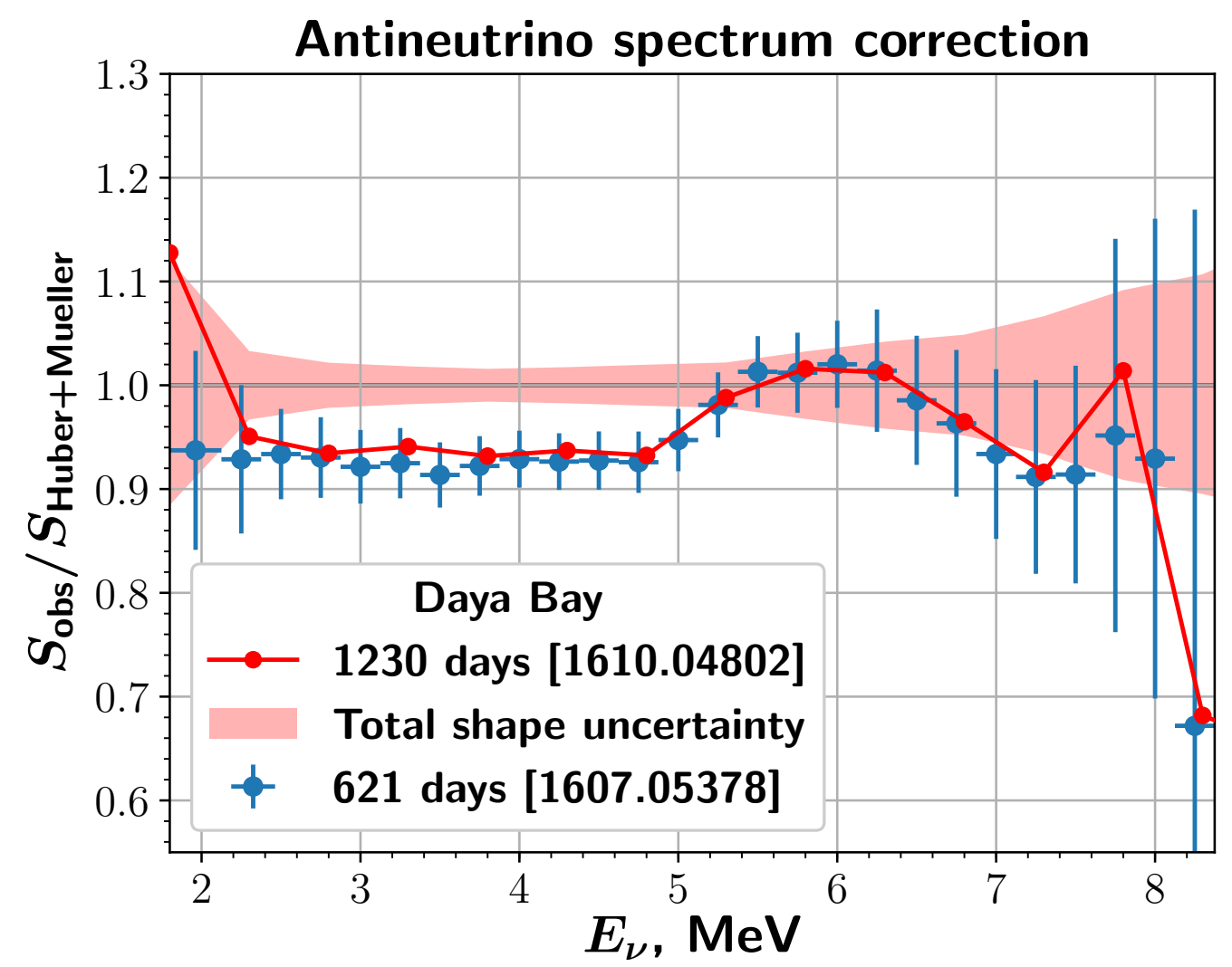

Figure 6: Ratio of measured spectrum to the prediction using Huber+Mueller model as a function of neutrino energy $E_{v}$. This ratio (red point) is fitted simultaneously with oscillation parameters [12]. Shaded area corresponds to the total uncertainty. Blue points with error bars are results of a dedicated analysis of $\bar{v}_{e}$ energy spectrum.

Multiple baselines and good energy resolution of the Daya Bay experiment allowed to test the sterile neutrino hypothesis. In Fig. 7 we display excluded regions in the plane of $\sin ^{2} 2 \theta_{14}, \Delta m_{41}^{2}$ based on analysis of data from Bugey-3 [29], Daya Bay [30] and their combination. Daya Bay and MINOS experiments, accounting for Bugey-3 data, performed also the combined analysis to probe regions allowed by the LSND and MiniBooNE experiments. Stringent limits on $\sin ^{2} 2 \theta_{\mu e} \equiv$ $4\left|V_{e 4}\right|^{2}\left|V_{\mu 4}\right|^{2}$ are set over six orders of magnitude over $\Delta m_{41}^{2}$ as displayed in Fig. 8. The sterileneutrino phase space allowed by LSND and MiniBooNE experiments is excluded for $\Delta m_{41}^{2}<0.8$ $\mathrm{eV}^{2}$ at $90 \%$ C.L.

The hypothesis of sterile neutrino is also disfavored by a dedicated Daya Bay analysis of reactor fuel evolution [31]. It was found that the deficit in IBD event rate can be primarily attributed to ${ }^{235} \mathrm{U}$ isotope which showed $7.8 \%$ smaller contribution with respect to Huber+Muler model, while the sterile neutrino hypothesis requires equal fractional deficit of each isotope's contribution.

\section{Conclusions}

In three neutrino-model most precise results are obtained for $\sin ^{2} 2 \theta_{13}$ and $\Delta m_{32}^{2}$ parameters 


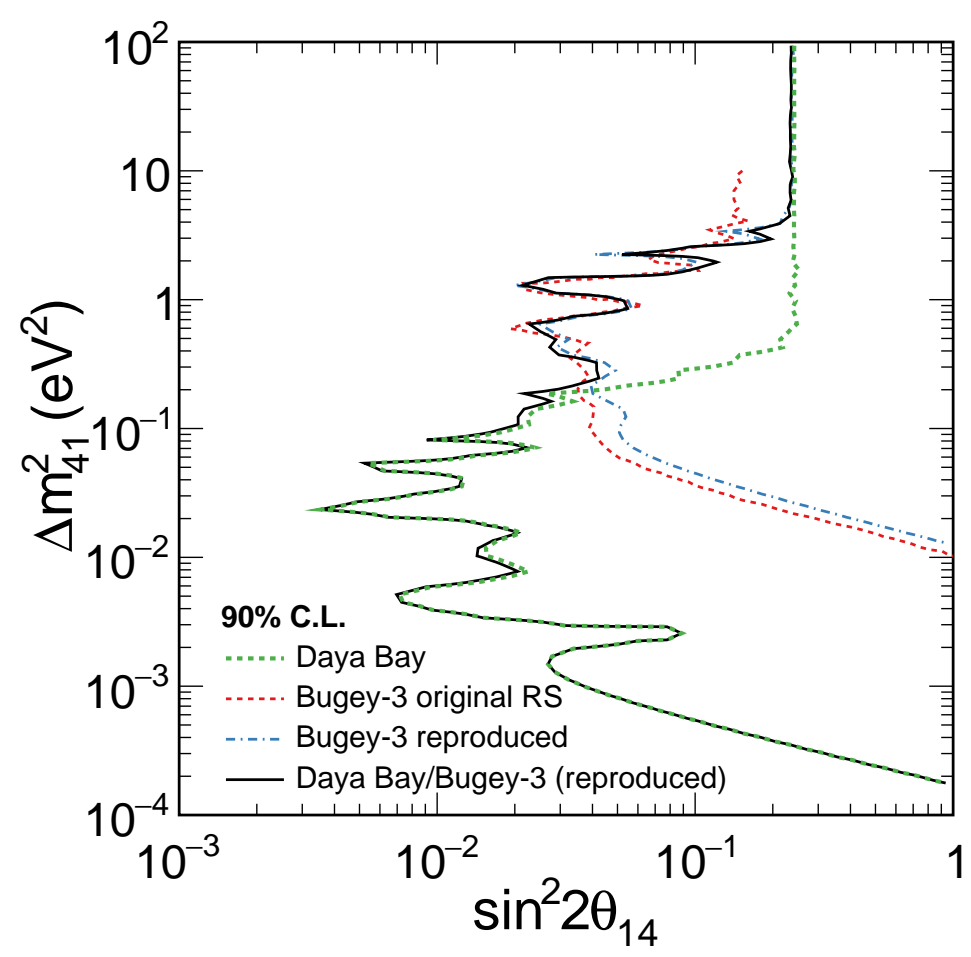

Figure 7: Excluded regions for the original Bugey-3 raster scan (RS) result (red line) [29], for the reproduced Bugey-3 with adjusted fluxes (blue line), for the Daya Bay result (green line) [30], and for the combined Daya Bay and reproduced Bugey-3 results (black line). The region to the right of the curve is excluded at the $90 \% \mathrm{CL}_{s}$.

based on 1230 days of data. The accuracy of these parameters are expected to reach about $3 \%$ by the end of data taking (2020). Updated independent $\mathrm{nH}$ rate-only analysis found values of these parameters consistent with $n G d$ analysis. A combined $n H+n G d$ analysis yields $\sin ^{2} 2 \theta_{13}=$ $0.082 \pm 0.004$.

The data was also analyzed within the model of neutrino wave packet setting up an upper limit on the intrinsic relative dispersion of neutrino momentum $\sigma_{\text {rel }}<0.20$ at a $95 \%$ C.L.

Reactor antineutrino flux shows an overall $\sim 5 \%$ deficit with an almost flat deficit of measured events for $E_{v}<5 \mathrm{MeV}$ and an agreement with the Huber+Muller model for $5.5 \mathrm{MeV} \lesssim E_{v} \lesssim 6.5$ $\mathrm{MeV}$.

A combined analysis of Daya Bay, Bugey-3 and MINOS data excludes most of the allowed by LSND and MiniBooNE experiments sterile-neutrino phase space for $\Delta m_{41}^{2}<0.8 \mathrm{eV}^{2}$ at 90\% C.L.

\section{References}

[1] F. P. An et al. [Daya Bay Collaboration], arXiv:1708.01265 [physics.ins-det].

[2] F. P. An et al. [Daya Bay Collaboration], Phys. Rev. Lett. 108, 171803 (2012) doi:10.1103/PhysRevLett.108.171803 [arXiv:1203.1669 [hep-ex]]. 


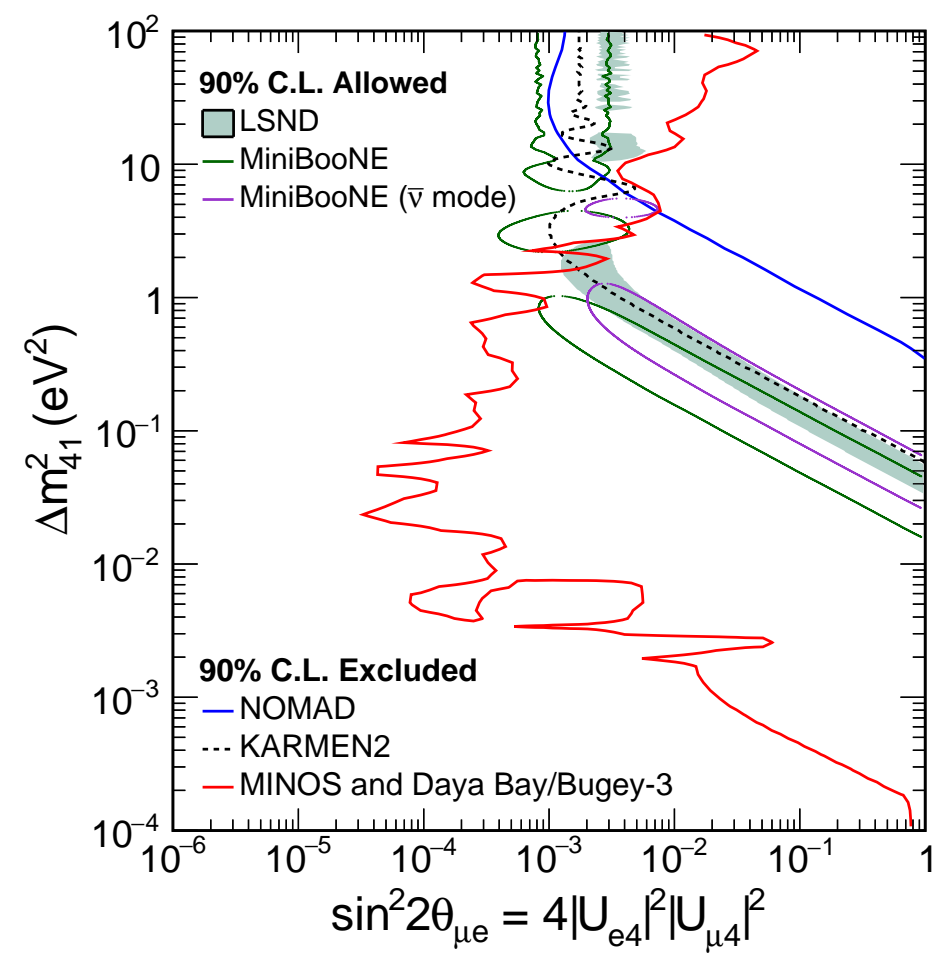

Figure 8: MINOS and Daya Bay + Bugey-3 combined $90 \% \mathrm{CL}_{s}$ limit on $\sin ^{2} 2 \theta_{\mu e}$ compared to the LSND and MiniBooNE 90\% CL allowed regions. Regions of parameter space to the right of the red contour are excluded. The regions excluded at $90 \% \mathrm{CL}$ by the KARMEN2 Collaboration [45] and the NOMAD Collaboration [46] are also shown.

[3] F. P. An et al. [Daya Bay Collaboration], Phys. Rev. Lett. 115, no. 11, 111802 (2015) doi:10.1103/PhysRevLett.115.111802 [arXiv:1505.03456 [hep-ex]].

[4] F. P. An et al. [Daya Bay Collaboration], Nucl. Instrum. Meth. A 685, 78 (2012) doi:10.1016/j.nima.2012.05.030 [arXiv:1202.6181 [physics.ins-det]].

[5] F. P. An et al. [Daya Bay Collaboration], Nucl. Instrum. Meth. A 773, 8 (2015) doi:10.1016/j.nima.2014.09.070 [arXiv:1407.0275 [physics.ins-det]].

[6] F. P. An et al. [Daya Bay Collaboration], Nucl. Instrum. Meth. A 811, 133 (2016) doi:10.1016/j.nima.2015.11.144 [arXiv:1508.03943 [physics.ins-det]].

[7] F. P. An et al. [Daya Bay Collaboration], Phys. Rev. Lett. 112, 061801 (2014) doi:10.1103/PhysRevLett.112.061801 [arXiv:1310.6732 [hep-ex]].

[8] F. P. An et al. [Daya Bay Collaboration], Phys. Rev. D 90, no. 7, 071101 (2014) doi:10.1103/PhysRevD.90.071101 [arXiv:1406.6468 [hep-ex]].

[9] F. P. An et al. [Daya Bay Collaboration], Phys. Rev. Lett. 113, 141802 (2014) doi:10.1103/PhysRevLett.113.141802 [arXiv:1407.7259 [hep-ex]].

[10] F. P. An et al. [Daya Bay Collaboration], Phys. Rev. Lett. 116, no. 6, 061801 (2016) Erratum: [Phys. Rev. Lett. 118, no. 9, 099902 (2017)] doi:10.1103/PhysRevLett.116.061801, 10.1103/PhysRevLett.118.099902 [arXiv:1508.04233 [hep-ex]]. 
[11] F. P. An et al. [Daya Bay Collaboration], Phys. Rev. D 93, no. 7, 072011 (2016) doi:10.1103/PhysRevD.93.072011 [arXiv:1603.03549 [hep-ex]].

[12] F. P. An et al. [Daya Bay Collaboration], Phys. Rev. D 95, no. 7, 072006 (2017) doi:10.1103/PhysRevD.95.072006 [arXiv:1610.04802 [hep-ex]].

[13] K. A. Olive et al. [Particle Data Group], Chin. Phys. C 38, 090001 (2014). doi:10.1088/1674-1137/38/9/090001

[14] J. H. Choi et al. [RENO Collaboration], Phys. Rev. Lett. 116, no. 21, 211801 (2016) doi:10.1103/PhysRevLett.116.211801 [arXiv:1511.05849 [hep-ex]].

[15] E. K. Akhmedov and A. Y. Smirnov, Phys. Atom. Nucl. 72, 1363 (2009) doi:10.1134/S1063778809080122 [arXiv:0905.1903 [hep-ph]].

[16] C. Giunti and C. W. Kim, Oxford, UK: Univ. Pr. (2007) 710 p

[17] E. K. Akhmedov and J. Kopp, JHEP 1004, 008 (2010) Erratum: [JHEP 1310, 052 (2013)] doi:10.1007/JHEP04(2010)008, 10.1007/JHEP10(2013)052 [arXiv:1001.4815 [hep-ph]].

[18] D. V. Naumov and V. A. Naumov, J. Phys. G 37, 105014 (2010) doi:10.1088/0954-3899/37/10/105014 [arXiv:1008.0306 [hep-ph]].

[19] P. Huber, Phys. Rev. C 84, 024617 (2011) Erratum: [Phys. Rev. C 85, 029901 (2012)] doi:10.1103/PhysRevC.85.029901, 10.1103/PhysRevC.84.024617 [arXiv:1106.0687 [hep-ph]].

[20] T. A. Mueller et al., Phys. Rev. C 83, 054615 (2011) doi:10.1103/PhysRevC.83.054615 [arXiv:1101.2663 [hep-ex]].

[21] P. Vogel, G. K. Schenter, F. M. Mann and R. E. Schenter, Phys. Rev. C 24, 1543 (1981). doi:10.1103/PhysRevC.24.1543

[22] A. Aguilar-Arevalo et al. [LSND Collaboration], Phys. Rev. D 64, 112007 (2001) doi:10.1103/PhysRevD.64.112007 [hep-ex/0104049].

[23] A. A. Aguilar-Arevalo et al. [MiniBooNE Collaboration], Phys. Rev. Lett. 110, 161801 (2013) doi:10.1103/PhysRevLett.110.161801 [arXiv:1303.2588 [hep-ex]].

[24] A. A. Aguilar-Arevalo et al. [MiniBooNE Collaboration], Phys. Rev. Lett. 98, 231801 (2007) doi:10.1103/PhysRevLett.98.231801 [arXiv:0704.1500 [hep-ex]].

[25] G. Mention, M. Fechner, T. Lasserre, T. A. Mueller, D. Lhuillier, M. Cribier and A. Letourneau, Phys. Rev. D 83, 073006 (2011) doi:10.1103/PhysRevD.83.073006 [arXiv:1101.2755 [hep-ex]].

[26] W. Hampel et al. [GALLEX Collaboration], Phys. Lett. B 420, 114 (1998). doi:10.1016/S0370-2693(97)01562-1

[27] J. N. Abdurashitov et al. [SAGE Collaboration], Phys. Rev. C 80, 015807 (2009) doi:10.1103/PhysRevC.80.015807 [arXiv:0901.2200 [nucl-ex]].

[28] V. A. Bednyakov, D. V. Naumov and O. Y. Smirnov, Phys. Usp. 59, no. 3, 225 (2016). doi:10.3367/UFNe.0186.201603b.0233

[29] Y. Declais et al., Nucl. Phys. B 434, 503 (1995). doi:10.1016/0550-3213(94)00513-E

[30] F. P. An et al. [Daya Bay Collaboration], Phys. Rev. Lett. 117, no. 15, 151802 (2016) doi:10.1103/PhysRevLett.117.151802 [arXiv:1607.01174 [hep-ex]].

[31] F. P. An et al. [Daya Bay Collaboration], Phys. Rev. Lett. 118, no. 25, 251801 (2017) doi:10.1103/PhysRevLett.118.251801 [arXiv:1704.01082 [hep-ex]]. 